\title{
Lilly Research Award Program (LRAP): A Successful Academia-Industry Partnership Model in the Context of Flow Chemistry for Drug Discovery
}

\author{
Carlos Mateos*
}

\begin{abstract}
The Lilly Research Award Program (LRAP) provides academic researchers worldwide with a gate to partner with Lilly internal scientists who are working on basic and applied research to collaboratively advance novel impactful projects. The pre-competitive nature of these projects is the most relevant feature as it permits the shared publication of the research outcomes immediately. In this article, this highly successful initiative is reviewed in the context of general academia-industry collaborations and the lessons learned from different shared projects, in the area of innovative continuous flow chemistry, will be discussed.
\end{abstract}

Keywords: Academia-Industry collaboration - Continuous flow chemistry · Eli Lilly and Co. · LRAP .

Pre-competitive research

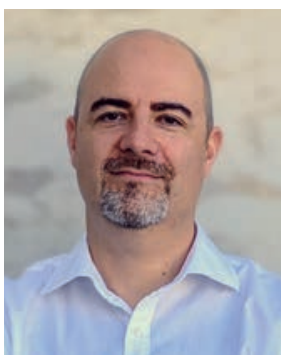

Dr. Carlos Mateos obtained his degree in Organic Chemistry, in 1998, in the University of Oviedo (Spain). Then, he moved to Leverkusen, (Germany) to enjoy an industrial internship in Bayer AG working on the industrial development of azodyes. In 1999, he moved back to Spain to pursue a $\mathrm{PhD}$ on natural product synthesis, in the University of Oviedo under the supervision of Professor José Barluenga. After receiving his $\mathrm{PhD}$ in 2004, Carlos joined the CRO Galchimia (Santiago de Compostela, Spain) as a project manager. In 2006, he joined Lilly where he is currently Research Scientist of the flow chemistry group in the R\&D Lilly site in Alcobendas (Madrid). His main research interests include medicinal chemistry, process development, continuous processing and external R\&D collaboration.

\section{Introduction}

R\&D in today's world is about networking, sharing and collaborating, especially in the area of new technologies. Access to the global knowledge in a particular field is not possible unless a good network of collaborators/partners (including academic and industrial) is built. Research collaboration between academic institutions and pharmaceutical companies are recognized as a great source of innovation and an essential way to advance science in drug discovery. ${ }^{[1]}$ Academic scientists bring excellent in-depth expertise and extensive basic research data to the table, whereas company researchers contribute with practical applicability in mind. This cooperation however is full of challenges. ${ }^{[2]}$ The main issues are usually both the technology transfer from academia to industry and the publication strategy. Traditionally, academic discoveries are valued for the level of the publications that arise from the research (impact factors) whereas for pharma, the value added normally requires ownership of the intellectual property rights.

\footnotetext{
${ }^{*}$ Correspondence: Dr. C. Mateos

E-mail: c.mateos@lilly.com

Centro de Investigación Lilly S.A., Avda. de la Industria, 30,

Alcobendas-Madrid 28108, Spain.
}

\subsection{Lilly Research Award Program}

The Lilly Research Award Program (LRAP) was born in 2011 to provide Lilly's best scientists who are working on basic and applied research with a gate to collaborate with global external researchers to advance research projects in partnership. These research projects range in focus, depending on the scientific discipline and may include validation of disease targets or biomarkers, new approaches to improve existing preclinical models or more basically, development of new models, methods or techniques. The key feature of LRAP is its pre-competitive character. Peer-reviewed publications and external scientific presentations at international meetings of the collaborative research are the main expected outcomes, but if the work developed constitutes an invention and a patent ultimately results, a free non-exclusive license could be extended. For a typical LRAP submission, a Lilly scientist sponsoring the project and an external investigator prepare a research proposal collectively, according to guidelines pre-established by Lilly. The proposals are reviewed by a panel of Lilly's top scientists and prioritized for funding based on the following considerations:

- Novelty and potential impact of proposed research.

- Expertise of the external investigator on the subject of study.

- Feasibility of the proposal and integration of both internal and external work plans.

Then, funding prioritization decisions, including written feedback on the proposal, will be made available to the internal Lilly sponsor. If the proposal is not considered for funding in that round, the internal sponsor is given a new opportunity, once he/she has addressed the reviewers' concerns. Since the initiation of the program in 2011, almost 600 proposals have been reviewed in ca. 15 funding rounds and about 140 have been fully funded so far, constituting a commitment that amounts to $>\$ 35 \mathrm{M}$ to date. Proposals have been submitted from all of Lilly's key R\&D sites and all therapeutic and functional areas from early drug discovery to development, including regulatory and global health outcomes are represented. More than 80 institutions, from 13 different countries, have been engaged in LRAP agreements. ${ }^{[3]}$ 
Apart from fostering innovation, other main goal of LRAP is cultivating professional relationships with worldwide experts, always as part of the development plan of internal Lilly scientists. This is especially important in areas such as new synthetic technologies where innovation starts mainly in academic labs co-developing, prototyping and testing new equipment and applications. In a typical LRAP project, experiments are usually conducted at the collaborator academic laboratory with Lilly scientists contributing mainly with data analysis, project planning and other virtual activities. Additionally, LRAP allows internal scientists to work with academic researchers on high-risk basic research projects that sometimes find difficult fit in internal workplans, due mainly to time constraints, but contributes largely to the internal scientist development and expertise which can afterwards be leveraged in portfolio projects.

\section{Successful LRAPs in the Context of Continuous Flow Chemistry Basic Research}

\subsection{Continuous-Flow Photochemistry}

Early on we became interested in applying photochemistry processes in continuous flow. The main reason for that is the efficiency and scalability that flow brings to these protocols. It is well known that photon transport is attenuated with the distance from the light source and this prevents the use of large vessels for scale-up in batch (Lambert-Beer law, Eqn. (1), Fig. 1). ${ }^{[4]}$

$$
\mathrm{A}=\log _{10} \mathrm{~T}=\log _{10} I_{\delta} I=\varepsilon c l
$$

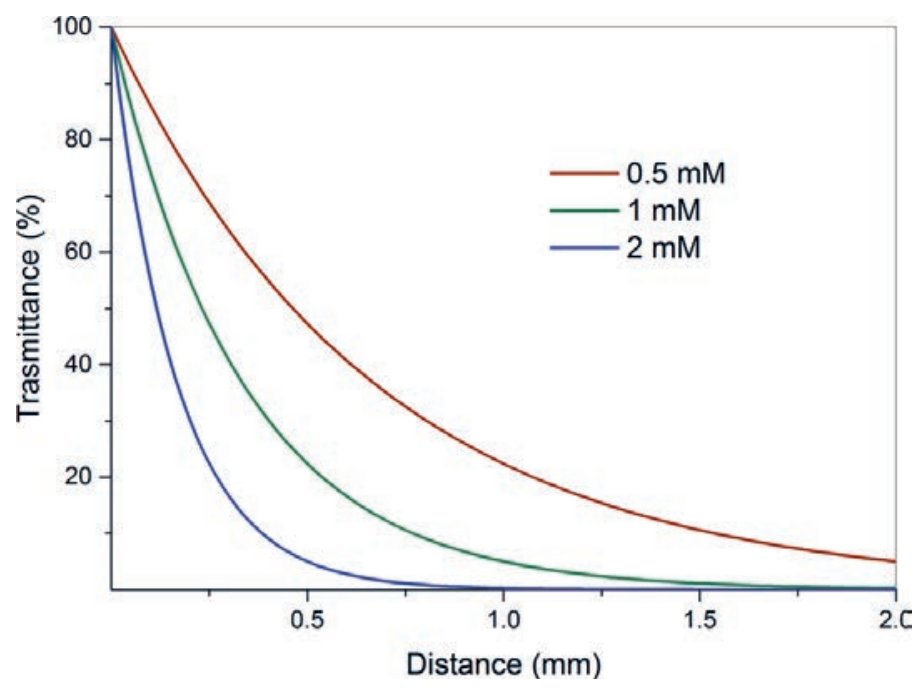

Fig. 1. Transmission of light as a function of distance in a photocatalytic reaction using $\mathrm{Ru}(\mathrm{bpy})_{3} \mathrm{Cl}_{2}$ (c $=0.5,1$, and $2 \mathrm{mM}, \varepsilon=13000 \mathrm{~cm}^{-1} \mathrm{M}^{-1}$ ) utilizing the Lambert-Beer correlation. Reproduced from ref. [6a] with permission of the American Chemical Society.

Booker-Milburn et al. published in 2005 an elegant solution to this scalability issue, utilizing flow chemistry in a simple setup (Fig. 2), for maximizing photo-efficiency. ${ }^{[5]}$

Later, many variations of that original scheme have been used for translating batch-to-flow photochemical processes or directly discover new ones. ${ }^{[6]}$

In 2012, we initiated a collaboration with Prof. Oliver Kappe's research group ${ }^{[7]}$ at the University of Graz in Austria for the development of photochemical applications in continuous flow.

\subsubsection{Benzylic Brominations}

At the beginning of our collaboration, we were very interested in developing an efficient and eco-friendly benzylic bro-

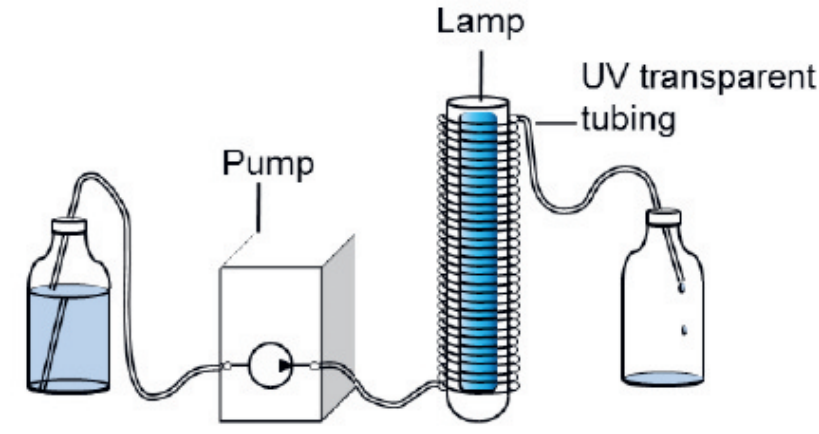

Fig. 2. Schematic of a typical macroflow photochemical reactor. Reproduced from ref. [6b] with permission of Beilstein-Institut.

mination protocol (the classical Wohl-Ziegler bromination under photochemical conditions). Many protocols describe the use of chemical radical initiators combined with NBS. ${ }^{[8]}$ However, we had encountered some challenges scaling up this process in several internal projects ${ }^{[9]}$ so we envisioned continuous flow as a perfect tool for addressing potential future needs. The ideal setup would be a simple system that allowed for the use of different light sources (in terms of wavelengths and wattage) and that could be reproduced easily in our labs. Prof. Kappe's group took on the challenge and we collaboratively designed a custom flow photoreactor that met all these criteria and demonstrated high flexibility (Fig. 3).[10]

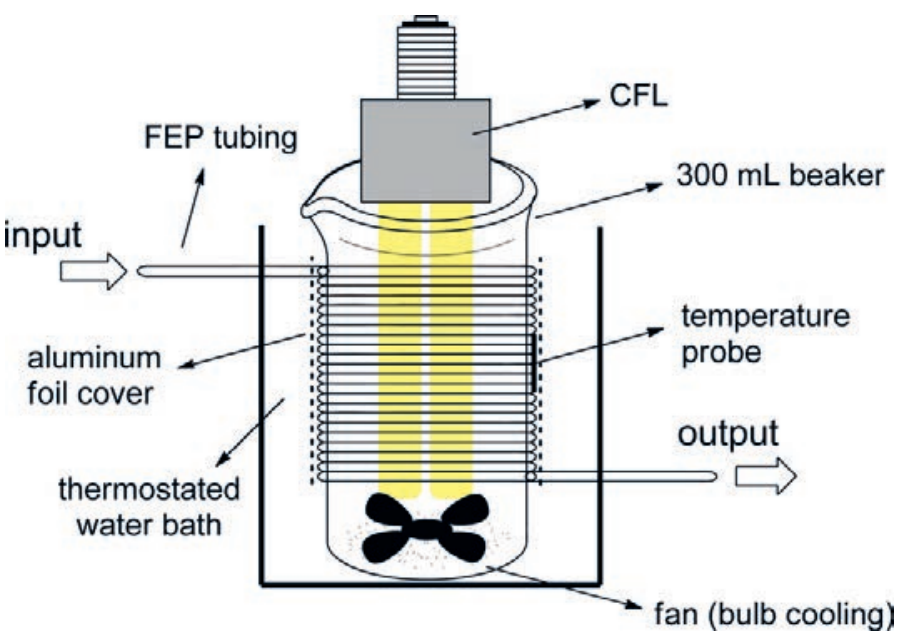

Fig. 3. Schematic diagram of the flow setup employed for the continuous-flow photochemical benzylic brominations. Reproduced from ref. [10] with permission of the American Chemical Society.

Once we had a suitable reactor system, we began the study of the photochemical radical bromination of the model system 4-tert-butyltoluene. Variables such as solvent (acetonitrile, THF), CFL-type ${ }^{[11]}$ light sources (30 W cool-white lamp, $15 \mathrm{~W}$ plantgrowing lamp, $25 \mathrm{~W}$ black light lamp), residence time and temperature were screened. Acetonitrile and black light were the best choices, being the ideal temperature variable depending on the reactivity of the substrate. Temperatures of $40{ }^{\circ} \mathrm{C}$ or $60^{\circ} \mathrm{C}$ were needed for unreactive substrates but lower temperatures such as $0{ }^{\circ} \mathrm{C}$ were used for the more reactive ones to avoid di-bromination by-products. The process showed general applicability for electron-poor toluene derivatives and other neutral benzylic scaffolds whereas high electron-rich toluene derivatives performed poorly under these conditions (Fig. 4).

To further demonstrate the scalability of the process, a scaleup version of this reactor with a $100 \mathrm{~W}$ black light lamp and a 28 $\mathrm{mL}$ FEP coiled reactor was designed, increasing the throughput 


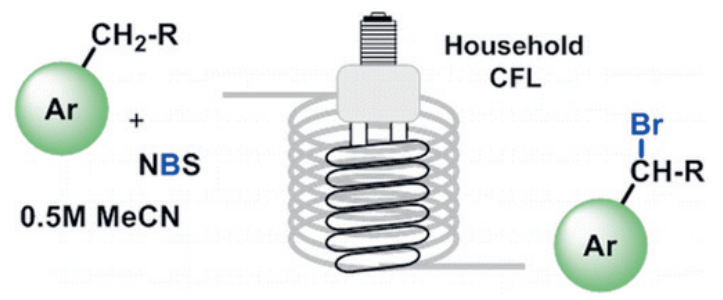

Fig. 4. Radical photochemical benzylic bromination in continuous flow. Reproduced from ref. [10] with permission of the American Chemical Society.

of the desired brominated products up to $38 \mathrm{~g} \mathrm{~h}^{-1}$ and exemplified for the case of 1-bromo-1-phenylacetone.

The protocol therein described was later proven to be completely reproducible; during the course of portfolio intermediate preparation in our labs several multigram batches of different brominated scaffolds have been furnished following it.

\subsubsection{Benzylic Fluorinations}

In general, toxic and highly reactive reagents are usually utilized in halogenation processes. Moreover, in many cases, selectivity is the major issue. Continuous flow chemistry can be a great advantage in these cases due to the reduced reactor volumes and excellent mass and heat transfer. ${ }^{[12]}$

Using a similar reactor design, previously shown for the bromination project, we developed a light-induced benzylic fluorination protocol. We chose to use Selectfluor as the fluorinating agent and after piloting the use of several bi-aryls as organic inexpensive photocatalysts and several light sources, we came to the conclusion that the combination of black-light (approx. $365 \mathrm{~nm}$ ) and xanthone as photo-organocatalyst was the best performer (Fig. 5).[13] For the less reactive substrates, the temperature had to be tuned up but high conversions were obtained in most of the substrates tested in 28 minutes residence time, using $5 \mathrm{~mol} \%$ xanthone in acetonitrile as solvent, including the biologically active compounds ibuprofen methyl ester and celestolide.

\subsubsection{Ketone Trifluoromethylations}

Later, during our LRAP project with Prof. Kappe's research group, we turned our attention to other interesting $\mathrm{C}-\mathrm{C}$ bond formation processes mediated by light, from the point of view of drug discovery. Trifluoromethylation reactions are very important in medicinal chemistry. ${ }^{[14]}$ In this particular sub-project and using a similar photochemical reactor setup, we developed a continuous flow procedure for the two-step preparation of $\alpha$-trifluoromethyl ketones. ${ }^{[15]}$ In the first step, the ketones were converted into their silyl enol ether derivatives by reaction with TMSOTf and diisopropylethylamine $\left(i \mathrm{Pr}_{2} \mathrm{EtN}\right)$ as the base, in flow. The silyl enol ethers generated in situ were then subjected to a visible lightinduced trifluoromethylation, combining $\mathrm{CF}_{3} \mathrm{SO}_{2} \mathrm{Cl}$ (triflyl chloride) as a source of $\mathrm{CF}_{3}$ radicals and Eosin $\mathrm{Y}$ as an inexpensive,

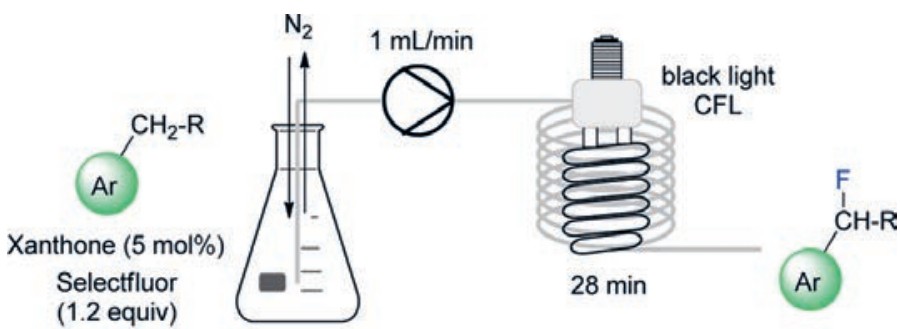

$\operatorname{MeCN}(0.1 \mathrm{M})$

Fig 5. Schematic diagram of the continuous-flow setup employed for the light-induced fluorination of benzylic compounds. Reproduced from ref. [13] with permission of the American Chemical Society.

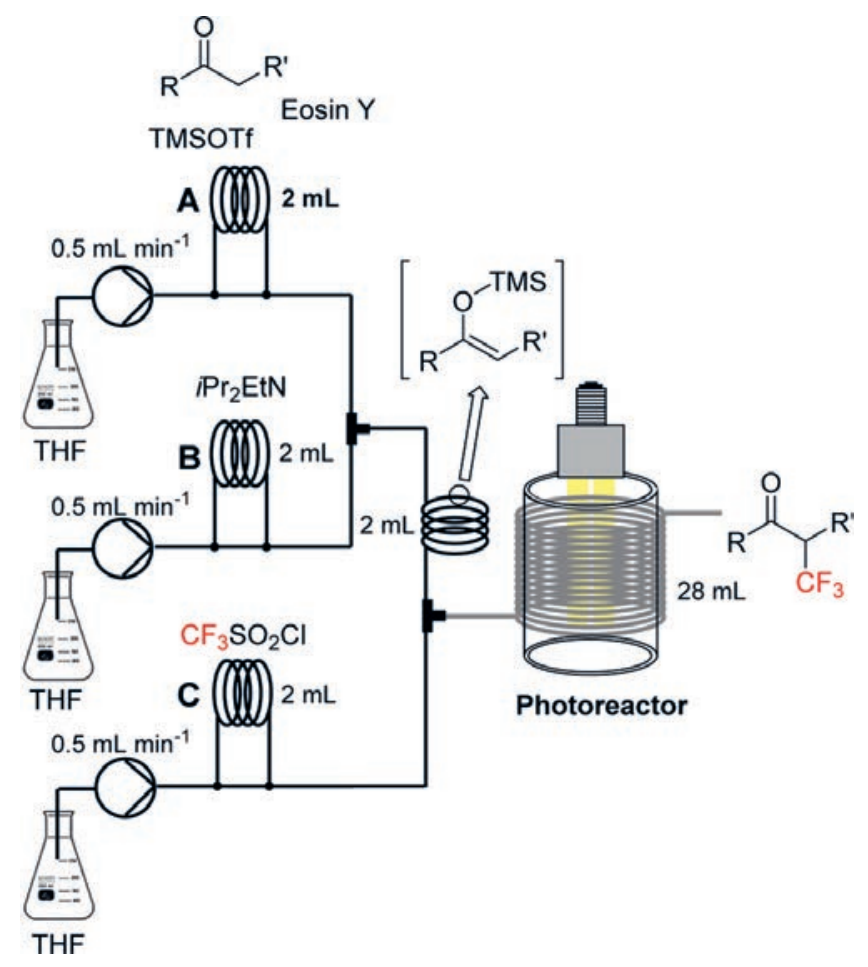

Scheme 1. Continuous flow setup for the two-step visible light induced $\alpha$-trifluoromethylation of ketones. Feed A: ketone (0.75 M), TMSOTf (1.5 equiv), and Eosin $\mathrm{Y}(0.5 \mathrm{~mol} \%)$ in THF. Feed $B$ : $i \operatorname{Pr}_{2}$ EtN (1.5 equiv) in THF. Feed C: $\mathrm{CF}_{3} \mathrm{SO}_{2} \mathrm{Cl}$ (1.5 equiv) in THF. Reproduced from ref. [15] with permission of the American Chemical Society.

nontoxic and readily available photoredox catalyst (Scheme 1). The two-step process requires only 20 min for completion, is run using THF as solvent and has been successfully tested for a variety of substrates.

\subsubsection{C-H Arylation of Heteroarenes}

$\mathrm{Bi}$ (hetero)aryls are very privileged scaffolds with a prominent presence in APIs, agrochemicals, and organic materials. ${ }^{[16]}$ In this program, we developed a novel light-induced, catalyst-free, $\mathrm{C}-\mathrm{H}$ arylation methodology for the preparation of bi(hetero)aryls from the direct reaction of anilines with arenes and heteroarenes. The anilines were reacted with tert-butylnitrite and transformed to nitrosamines which, in equilibrium with their corresponding diazo anhydrides, decomposed under light irradiation at room temperature. In the presence of (hetero)arenes, this radical fragmentation results in the formation of bi(hetero)aryls, producing $\mathrm{N}_{2}$ gas, $\mathrm{H}_{2} \mathrm{O}$, and $t \mathrm{BuOH}$ as the only byproducts. Additionally, in order to make this method safely scalable, a continuous flow procedure was implemented. In this way, hazardous reactive intermediates like nitrosamine and diazo-type species are generated in situ and are immediately consumed within the reactor, avoiding the han-

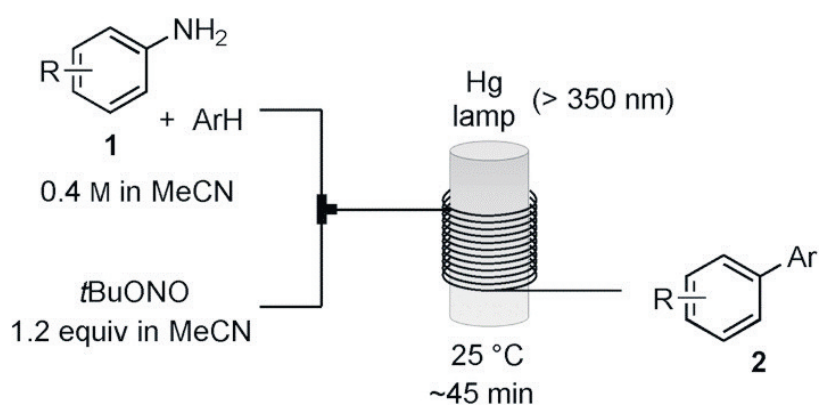

Scheme 2. Biaryls from anilines under photochemical reaction conditions in continuous flow. Reproduced from ref. [17] with permission of John Wiley and Sons. 
dling of these substances. The continuous flow process involved a residence time of $45 \mathrm{~min}$ at room temperature and was run in acetonitrile as solvent. ${ }^{[17]}$

In this project, we used the commercially available Vapourtec photoreactor (UV-150 E Series), which consisted of transparent (FEP) tubing ( $1 \mathrm{~mm}$ i.d., $10 \mathrm{~mL}$ volume) coiled around a poweradjustable medium pressure $\mathrm{Hg}$ lamp (max.150 W). ${ }^{[18]}$ The combination of this UV lamp with a long-pass filter that allowed to cut-off irradiation with wavelengths below $350 \mathrm{~nm}$, provided the best results (Scheme 2).

\subsection{Electrochemistry in Flow}

Continuous flow electrochemistry, like the previously discussed photochemistry procedures, offers many advantages over analogous batch conditions. ${ }^{[19]}$ Both can be considered as 'reagent-less' reactions with advantages in safety, product purification and environmental footprint of the process. In contrast to a photochemical reaction where photons activate molecules, in an electrochemical process the reaction is facilitated by electrons (oxidation or reduction processes) and flow electrochemical reactor setups are able to reduce the inter-electrode distance to allow for better electron transfer. Due to our keen interest in this disruptive technology, we partnered with Prof. Richard Brown's research group in the University of Southampton $(\mathrm{UK})^{[20]}$ in an another LRAP endeavor. This group had collaborated in the development of several flow electrochemical reactor devices, now commercially available in the market.[21]

In this LRAP project, we developed an electrochemical deprotection of PMB ( $p$-methoxybenzoyl) ethers in an undivided electrochemical flow reactor (Ammonite 8) ${ }^{[21 \mathrm{~b}]}$ and demonstrated the application of this process for a wide range of substrates (Scheme 3). ${ }^{[22]}$ Furthermore, the ease of scale-up in a laboratory setting was highlighted with the use of the larger reactor (Ammonite 15) (21b] $^{[2}$ yielding a throughput of $c a .20 \mathrm{~g} / \mathrm{h}$. The procedure developed used $\mathrm{Et}_{4} \mathrm{NBF}_{4}$ as recoverable electrolyte, $\mathrm{MeOH}$ as solvent and afforded good yields. Additionally, we also measured sustainability metrics (RME) ${ }^{[23]}$ that were favorably compared with those of other reported procedures for PMB deprotection.

In a related project, we analyzed the influence of the presence of non-ionic surfactants in extended channel, narrow gap flow electrolysis cells. We demonstrated in this work that the use of these additives (mainly Triton X-100 or Brij L23) to the electrolyte medium leads to a significant improvement to the current efficiency for the model methoxylation of $\mathrm{N}$-formylpyrrolidine, in an electrolytic flow cell. ${ }^{[24]}$

\subsection{Ruthenium-catalyzed Red-Ox Processes}

Recently, we collaborated within other LRAP project with Prof. Steven Ley's research group from the University of Cambridge. Prof. Steven Ley ${ }^{[25]}$ is one of the leading experts and a pioneer in the evolution of continuous flow chemistry for reagent immobilization, multi-step natural product synthesis and innovative applications such as online monitoring, lab automation, etc.

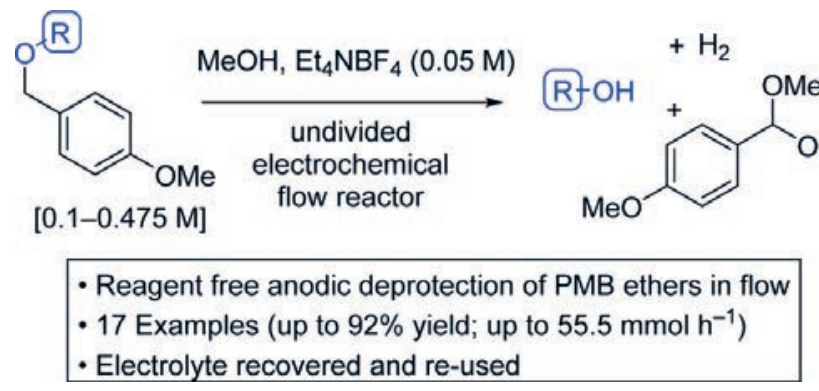

Scheme 3. Electrochemical PMB deprotection of alcohol in continuous flow. Reproduced from ref. [22] with permission of the American Chemical Society.

\subsubsection{Alcohol Oxidations Using Acetone as Eco-friendly Oxidant}

The first project we embarked on was the development of friendly conditions for general alcohol oxidation processes. The oxidation of alcohols is a fundamental transformation in chemical synthesis but it often requires toxic reagents, lacks selectivity and is challenging for scale-up. ${ }^{[26]}$ In this project, we utilized a hydrogen borrowing strategy, using very low loading of an affordable ruthenium catalyst for the Oppenauer type oxidation of secondary alcohols, using acetone as oxidant (and solvent) in very short residence times (4-40 $\mathrm{min}$ ) (Scheme 4). The system demonstrated high selectivity toward secondary over primary alcohols and was demonstrated to be scalable to multigram quantities. ${ }^{[27]}$ The substrate scope is very wide and it includes selectivity-compromised alkenes, heterocycles and esters. Pleasingly, very hindered alcohols could be successfully oxidized, as well as natural products such as $5 \alpha$-cholestan- $3 \beta$-ol and terfenadine. To our delight, this protocol has been used internally with great success on several occasions, in portfolio projects, where other standard batch oxidation conditions did not work satisfactorily.

\subsubsection{Arylnitrile Reductions Using Isopropanol as Eco-friendly Reductant}

In a subsequent work, we employed our red-ox catalyst (phosphine- and base-free) to promote the reduction of aromatic nitriles to the corresponding benzylic amines ${ }^{[28]}$ under hydrogen transfer conditions in continuous flow, in this case by using 2-propanol as both solvent and reducing agent. ${ }^{[29]}$ To enable a suitable downstream isolation we decided to obtain the primary amines as their $\mathrm{HCl}$ salts. The conditions allowed for the nitrile reduction in less than 10 min residence time, required no additives and worked with a fairly wide scope of arylnitriles including chloride derivatives, highlighting the selectivity of the developed process (Scheme 5).

\subsubsection{Ru-catalyzed Alcohol Aminations}

A third subproject, within the LRAP collaboration with Prof. Ley's group, involved the combination of both an oxidation and a reduction process using a similar catalytic system and consisted in the continuous amination of alcohols via hydrogen borrowing. [30] This transformation is usually performed by a substitution or reductive amination procedure, necessitating a two- or three-step

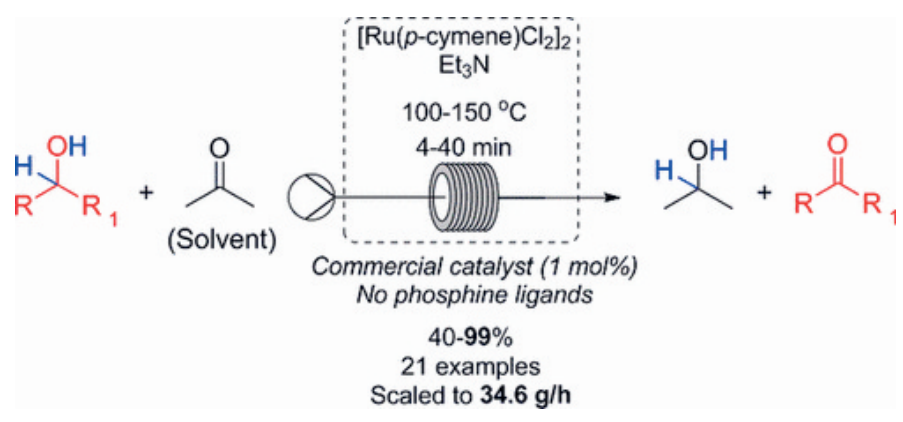

Scheme 4. Continuous flow alcohol oxidation under hydrogen borrowing conditions. Reproduced from ref. [27] with permission of the American Chemical Society.

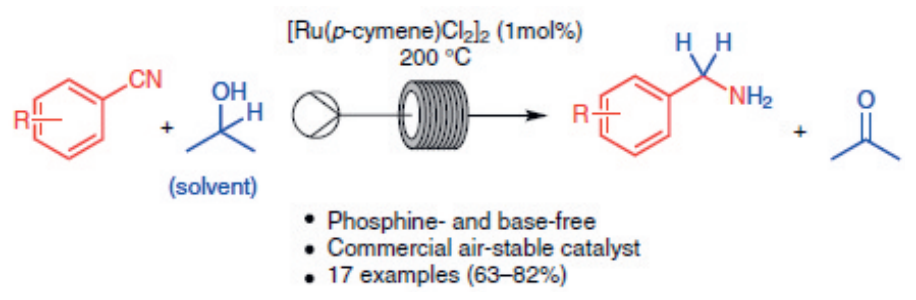

Scheme 5. Aromatic nitriles transfer hydrogenation in continuous flow. Reproduced from ref. [29] with permission of Thieme Publishing Group. 
process such as alcohol activation/amine displacement or alcohol oxidation/imine formation/imine reduction, usually using hazardous reagents. A hydrogen transfer approach represents excellent atom efficiency and generates only water as by-product during the overall conversion of alcohol to amine. It consisted of an oxidation phase to generate a carbonyl species using a suitable hydrogen atom catalytic carrier, followed by rapid imine formation and final return of hydrogen to effect formation of the product amine (Scheme 6). ${ }^{[31]}$

The method developed utilized a low loading $(0.5 \%)$ of a commercial catalyst system ([Ru(p-cymene $\left.) \mathrm{Cl}_{2}\right]_{2}$ and DPEPhos), reagent grade solvents and was selective for primary alcohols. The process takes place with a residence time of $10 \mathrm{~min}$ at $250{ }^{\circ} \mathrm{C}$ (Scheme 7).

The results from a brief reaction scope study indicated that it worked generally with primary alcohols selectively and accepted primary and secondary amines broadly. Interestingly, successful selective mono-methylation of amines using methanol in toluene was achieved under these conditions. To further demonstrate the scalability of the developed process, we used a larger coil reactor (35 mL), 1.6 equiv. benzyl alcohol and no solvent to produce 1.2 $\mathrm{kg}$ of product over $9 \mathrm{~h}$ of continuous processing time. The crude output was then downstream processed as its $\mathrm{HCl}$ salt, delivering

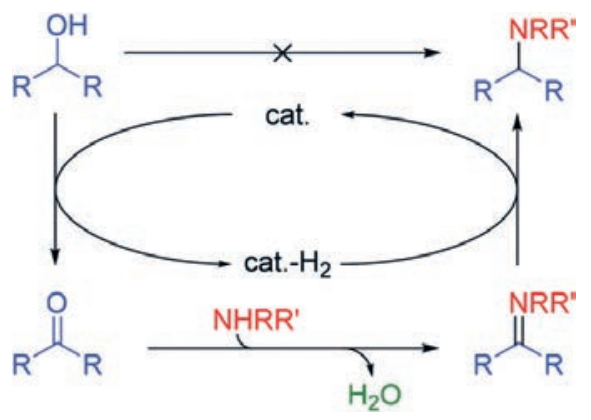

Scheme 6. Mechanism of the Alcohol amination via hydrogen borrowing. Reproduced from ref. [31] with permission of the Royal Society of Chemistry.

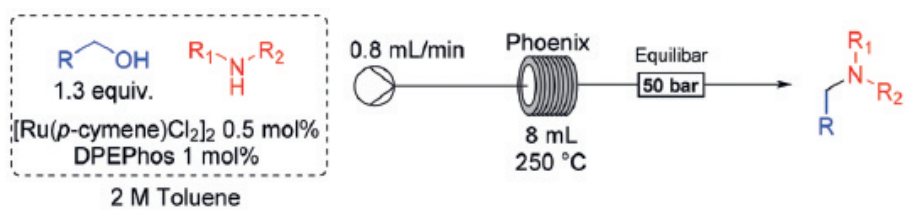

Scheme 7. Conditions for the alcohol amination process in continuous flow. Reproduced from ref. [31] with permission of the Royal Society of Chemistry.

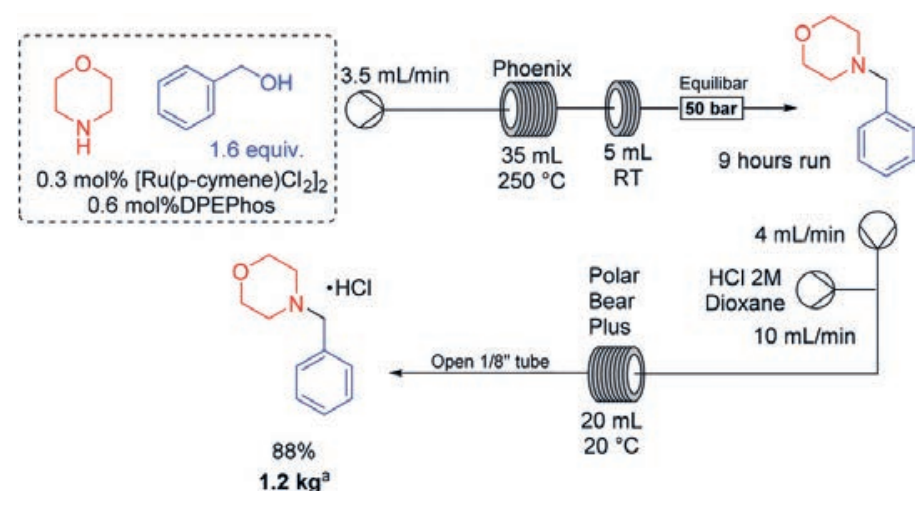

Scheme 8. Scale-up experiment. $500 \mathrm{~mL}$ of crude material from the first step (1.9 L over 9 hours) was processed in the downstream, value calculated back for the whole steady state collection. Reproduced from ref. [31] with permission of the Royal Society of Chemistry. the desired product clean of ruthenium $(<6 \mathrm{ppm})$ in $88 \%$ yield overall (Scheme 8).

\section{On-going and Future LRAPs in the Pipeline}

We have LRAP projects either on-going or in approved funding status to collaborate with Prof. David Macmillan (Princeton University, photoredox catalysis applications), Prof. Klavs Jensen (MIT, automation in continuous flow processes) and Prof. Ian Baxendale (Durham University, handling of solid materials in continuous flow chemistry). The outcomes of those projects will be published accordingly.

\section{Acknowledgements}

The author would like to thank Prof. Oliver Kappe, Prof. Richard Brown and Prof. Steven Ley and their respective research group members for the commitment and willingness to collaborate with us. In addition, the author thanks the Eli Lilly \& Company LRAP Operations group, Dr. Oscar de Frutos and Dr. Juan A. Rincón for helpful discussion during the manuscript preparation.

Received: May 20, 2019

[1] Recent publications on Pharma Industry-Academia collaborations: a) M. Palmer, R Chagurotu, Exp. Op. Drug Discov. 2017, 12, 537; b) J. S. Fogt, J. P. Kitzmiller, J. Transl. Sci. 2018, 4, 1.

[2] T. Gersdorf, V. F. He, A. Schlesinger, G. Koch, D. Ehrismann, H. Widmer, G. v. Krogh, Nat. Rev. Drug Discov. 2019, https://doi.org/10.1038/d41573-01900001-2.

[3] Eli Lilly and Co. shared communication.

[4] Y. Su, N. J. W. Straathof, V. Hessel, T. Noel, Chem. Eur. J. 2014, 20, 10562.

[5] B. D. A. Hook, W. Dohle, P. R. Hirst, M. Pickworth, M. B. Berry, K. I. Booker-Milburn, J. Org. Chem. 2005, 70, 7558.

[6] For interesting reviews on photochemistry in continuous flow see: a) D. Cambie, C. Bottecchia, N. J. W. Straathof, V. Hessel, T. Noel, Chem. Rev. 2016, 116, 10276; b) J. P. Knowles, L. D. Elliott, K. I. Booker-Milburn, Beilstein J. Org. Chem. 2012, 8, 2025.

[7] http://goflow.at/, accessed on 06/05/2019.

[8] For some recent examples, see: a) G. Haberhauer, C. Tepper, C. Wölper, D. Bläser, Eur. J. Org. Chem. 2013, 2325; b) D. T. Sreedharan, D. L. Clive, J. Org. Biomol. Chem. 2013, 11, 3128; c) V. R. Pallela, M. R. Mallireddigari, S. C. Cosenza, B. Akula, D. R. C. V. Subbaiah, E. P. Reddy, M. V. R. Reddy, Org. Biomol. Chem. 2013, 11, 1964; d) J. Chen, W. Sun, J. Yang, H. Sun, Z Wang, L. Dong, C. Xi. Qiao. Bioorg. Med. Chem. Lett. 2013, 23, 3785

[9] A. C. DeBaillie, C. D. Jones, N. A. Magnus, C. Mateos, A. Torrado, J. P. Wepsiec, R. Tokala, P. Raje, Org. Process Res. Dev. 2015, 19, 1568

[10] D. Cantillo, O. de Frutos, J. A. Rincon, C. Mateos, C. O. Kappe, J. Org. Chem. 2014, 79, 223.

[11] CFLs: household compact fluorescent lamps.

[12] For a review on halogenation reactions in flow see: D. Cantillo, C. O. Kappe, React. Chem. Eng. 2017, 2, 7.

[13] D. Cantillo, O. de Frutos, J. A. Rincon, C. Mateos, C. O. Kappe, J. Org. Chem. 2014, 79, 8486 .

[14] S. Barata-Vallejo, B. Lantaño, A. Postigo, Chem. Eur. J. 2014, 20, 16806.

[15] D. Cantillo, O. de Frutos, J. A. Rincon, C. Mateos, C. O. Kappe, Org. Lett. 2014, 16, 896

[16] a) C.-L. Sun, Z.-J. Shi, Chem. Rev. 2014, 114, 9219; b) I. Hussain, T. Singh, Adv. Synth. Catal. 2014, 356, 1661.

[17] D. Cantillo, O. de Frutos, J. A. Rincon, C. Mateos, C. O. Kappe, Chem. Eur. J. 2015, 21, 12894.

[18] https://www.vapourtec.com/products/flow-reactors/photochemistry-uv150-photochemical-reactor-features, accessed on 13/05/2019.

[19] For a recent review on electrochemistry in flow see: M. Atobe, H. Tateno, Y. Matsumura, Chem. Rev. 2018, 118, 4541 .

[20] https://www.southampton.ac.uk/chemistry/about/staff/rcb1.page?\#research, accessed on 07/05/2019.

[21] Commercially available electrochemistry flow reactors: a) Syrris FLUX: R. A. Green, R. C. D. Brown, D. Pletcher, J. Flow Chem. 2015, 5, 31; b) CRD (Cambridge Reaction design) Ammonite systems: R. A. Green, R. C. D. Brown, D. Pletcher, B. Harji, Electrochem. Comm. 2016, 73, 63. https:// www.cambridgereactordesign.com/ammonitel, accessed on 09/05/2019.

[22] R. A. Green, K. E. Jolley, A. A. M. Al-Hadedi, D. Pletcher, D. C. Harrowven, O. De Frutos, C. Mateos, D. J. Klauber, J. A. Rincón, R. C. D. Brown, Org. Lett. 2017, 19, 2050.

23] RME: Reaction Mass Efficiency. R. A. Sheldon, Chem Soc. Rev. 2012, 41, 1437. 
[24] A. A. Folgueiras-Amador, K. E. Jolley, P. R. Birkin, R. C.D. Brown, D. Pletcher, S. Pickering, M. Sharabi, O. de Frutos, C. Mateos, J. A. Rincón, Electrochem. Comm. 2019, 100, 6.

[25] https://en.wikipedia.org/wiki/Steven_V._Ley, accessed 08/05/2019.

[26] a) S. Caron, R. W. Dugger, S. G. Ruggeri, J. A. Ragan, D. H. B. Ripin, Chem Rev. 2006, 106, 2943; b) H. P. L. Gemoets, Y. Su, M. Shang, V. Hessel, R. Luque, T. Noel, Chem. Soc. Rev. 2016, 45, 83.

[27] R. Labes, C. Battilocchio, C. Mateos, G. R. Cumming, O. de Frutos, J. A Rincón, K. Binder, S. V. Ley, Org. Process Res. Dev. 2017, 21, 1419.
[28] D. B. Bagal, B. M. Bhanage, Adv. Synth. Catal. 2015, 357, 883.

[29] R. Labes, D. González-Calderón, C. Battilocchio, C. Mateos, G. R. Cumming, O. de Frutos, J. A. Rincón, S. V. Ley, Synlett 2017, 28, 2855.

[30] A. Corma, J. Navas, M. J. Sabater, Chem. Rev. 2018, 118, 1410.

[31] R. Labes, C. Mateos, C. Battilocchio, Y. Chen, P. Dingwall, G. R. Cumming, J. A. Rincón, M. J. Nieves-Remacha, S. V. Ley, Green Chem. 2019, 21, 59; highlighted in Synfacts 2019, 15, 211. 